\title{
Motivación: Buen desempeño laboral de los trabajadores de la gestión pública
}

\author{
Sandra Ruiz Corea \\ sruiz_correa@hotmail.com \\ Carlos Miguel Aguilar Saldaña \\ agua0122@hotmail.com \\ Hugo Olivas Salazar \\ holivassalazar@gmail.com \\ Janeth Ruiz Correa \\ Ruizcorreajaneth.72@gmail.com \\ Percy Flores Arévalo \\ Percyflores1976@gmail.com \\ Rosa Elena Cueto Orbe \\ Rosacueto.69@gmail.com \\ Escuela de post grado \\ Universidad César Vallejo \\ Tarapoto - Perú
}

\section{RESUMEN}

La investigación se caracteriza principalmente en establecer la relación entre la motivación y el desempeño laboral de los trabajadores. La justificación se basa en las afirmaciones de Robbins (2008), que la motivación es el proceso mediante el cual los esfuerzos de una persona se ven energizados, dirigidos y sostenidos hacia el logro de las metas y objetivos institucionales. El objetivo es determinar el grado de relación entre la motivación y el desempeño laboral de los trabajadores. Se recurrió al método lógico deductivo y al diseño de investigación no experimental correlacional. Como técnicas de recolección de datos se recurrió a la encuesta y como instrumento al cuestionario de preguntas. En tal sentido se obtuvo como resultado que el $50 \%$ de los colaboradores, presentaron niveles bajos de desempeño, debido a su falta de conocimiento sobre la totalidad de las actividades que se desarrollan dentro de su unidad o área de trabajo, o por emplear más recursos de los necesarios, y en el peor de los casos, por no cumplir con las metas establecidas por una falta de esfuerzo.

Palabras clave: Motivación; desempeño; eficiencia; eficacia. 


\title{
Motivation: Good job performance of public management workers
}

\begin{abstract}
The research is mainly characterized in establishing the relationship between motivation and work performance of workers. The justification is based on the assertions of Robbins (2008), That motivation is the process by which a person's efforts are energized, directed and sustained towards the achievement of institutional goals and objectives. The objective is to determine the degree of relationship between motivation and work performance of workers. The deductive logic method and the correlational non-experimental research design were used. The survey was used as data collection techniques and the questionnaire as an instrument. In this sense, it was obtained as a result that $50 \%$ of the collaborators presented low levels of performance, due to their lack of knowledge about all the activities that are developed within their unit or work area, or because of using more resources than necessary, and in the worst case, for not meeting the goals set due to a lack of effort.
\end{abstract}

Keywords: Motivation; performance; efficiency; effectiveness.

Artículo recibido: 05 de Mayo 2021 Aceptado para publicación: 20 de Junio 2021 Correspondencia: $\underline{\text { sruiz_correa@hotmail.com }}$ Conflictos de Interés: Ninguna que declarar 


\section{INTRODUCCIÓN}

En el área laboral es cada vez más importante conocer aquellos factores que repercuten en el desempeño de las personas. Se sabe que ciertos componentes físicos y sociales influyen sobre el comportamiento humano, es allí donde la motivación para el desempeño determina la forma en que el individuo percibe su trabajo, su rendimiento, su productividad, su satisfacción, entre otros aspectos.

Por lo tanto, la motivación y el desempeño laboral, reflejan los valores, actitudes y creencias de los miembros de la organización, constituyendo así la personalidad de la misma.

Siendo la motivación según (Chiavenato. 2000, p 6), "La voluntad de ejercer un esfuerzo persistente y de alto nivel a favor de las metas de la organización, donde el hombre es considerado un animal complejo" (p.6); es por ello que se considera que el individuo está dotado de necesidades complejas y diferenciadas; las cuales orientan y dinamizan el comportamiento. En este sentido, la opinión de Robbins (2004), es pertinente cuando afirma: Es por ello que la motivación es parte importante de la administración, ya que influye sobre las personas para que ejecuten tareas en bien de la organización. Según (Smith, citado por Cummings y Schwab 1985) expresa: “No es cuestión de que una mente calmada juzgue por las acciones externas, se debe fijar en la parte interior y ver qué resortes se ponen en movimientos, así que primero hay que conocer la naturaleza humana. Para lograr una verdadera motivación en el trabajador, debe haber una buena política y cultura organizacional en constante evolución que favorezca el desarrollo de los individuos en ese ambiente y permita obtener lo mejor de ellos, así como también permita que ellos se sientan satisfechos en el logro de sus aspiraciones personales, ya que como lo explica Marcano M. (2006): "La motivación del trabajador cambia de tiempo en tiempo" (p. 99) y las gerencias organizacionales no pueden descuidar este factor ni deben catalogarlo como un gasto; sino más bien como una inversión a la que se le puede sacar el mayor provecho posible.

Dentro de la UE-400-DIRES San Martin, se ha logrado identificar un deficiente y bajo nivel de desempeño en cuanto a los colaboradores, producto de ciertas actividades negativas, tomadas por el personal más antiguo, entre ellas, se evidencia la carencia de conocimientos, así como de habilidades respecto al trabajo u actividad que realizan, lo que individuo, así como en su conjunto. Por otro lado, aquellas personas que se sienten 
seguras de su trabajo, y que ya sienten que les falta poco para jubilarse, no muestran mayor compromiso con la institución, evidenciándose en inasistencias y tardanzas en determinados días.

Luego de analizar este comportamiento de forma detenida, se consideró que la causa más probable para que se suscitara estos problemas es la motivación, ya que en su mayoría los trabajadores, se quejan de la que perciben, de las condiciones en las que se encuentran, así como con las expectativas y logros que pudieron, pueden o podrán alcanzar con el trabajo.

Es, por consiguiente, necesario analizar la motivación y su relación con el desempeño laboral de las personas que laboran en las diferentes áreas de la UE-400-DIRES SM, debido a que la motivación entre compañeros de trabajo es un elemento importante que ejerce una influencia fuerte sobre actitudes y valores de éstos. Es necesario que en los departamentos de Administración y Recursos Humanos se defina cuáles son las necesidades laborales e individuales de los empleados, que coadyuven a la satisfacción en el trabajo, a fin de solventar aquellas que conduzcan a situaciones negativas o de insatisfacción que afecten el desempeño laboral.

La presente investigación trata de destacar la incidencia de la motivación en el desempeño laboral, con el propósito de obtener un resultado más efectivo del personal administrativo.

Ante este panorama se considera interesante responder la siguiente interrogante: ¿En qué medida la Motivación se relaciona con el Desempeño Laboral de los Trabajadores de la UE-400-DIRES SM 2014?

\section{Motivación}

Robbins (2008). La motivación se refiere al proceso mediante el cual los esfuerzos de una persona se ven energizados, dirigidos y sostenidos hacia el logro de una meta. Esta definición tiene tres elementos clave: energía, dirección y perseverancia.

Promover altos niveles de motivación en el desempeño laboral es un asunto organizacional importante, y los gerentes siguen buscando las respuestas.

Según Vidaurre (2009), considera que la motivación es un factor fundamental dentro de la organización, es por ello que debe de ser administrado de forma eficiente para evitar futuros conflictos. Sin embargo, se pudo constatar que la institución en estudio no destina de manera adecuada la administración de los incentivos y recompensas en el 
personal, esto genera aún más conflictos y por consiguiente una baja en la productividad porque se encuentran en su mayoría desmotivados.

Según. Robbins (2005), quien define a la motivación como el conjunto de procesos que inciden en la intensidad, dirección y persistencia del esfuerzo que realiza una persona para alcanzar un objetivo; esto indica que el personal no es más competente ni productivo porque no existe una fuerza interna (motivación intrínseca) ni externa (motivación extrínseca) que fomente su mayor esfuerzo y participación, esto genera que no se concreten ni direccionen los objetivos que se ha propuesto la institución.

\section{La teoría de la motivación: teoría de la jerarquía de las necesidades}

Abraham Maslow, psicólogo que propuso que dentro de cada persona existe una jerarquía de cinco necesidades:

1) Necesidades fisiológicas. Las necesidades de una persona de comida, bebida, refugio, sexo, y otros requerimientos físicos.

2) Necesidades de seguridad. Las necesidades de una persona de seguridad y protección ante el daño físico y emocional, así como la seguridad de que las necesidades físicas se seguirán cubriendo.

3) Necesidades sociales. Las necesidades de una persona de afecto, pertenencia, aceptación y amistad.

4) Necesidades de estima. Las necesidades de una persona de factores internos de estima, como el respeto por sí mismo, la autonomía y el logro, y los factores externos de estima, como el estatus, el reconocimiento y la atención.

5) Necesidades de autorrealización. Las necesidades de una persona de crecimiento, de lograr su potencial y de realización personal; la motivación para convenirse en lo que uno tiene la capacidad de llegar a ser.

Black (2010). Cuando utilizamos el término "motivación", independientemente de la situación, ¿qué significa? La motivación se define como un conjunto de fuerzas que impulsan, dirigen y mantienen cierta conducta. Tales fuerzas quizá provengan del interior de las personas, y se conocen como "empuje" de las fuerzas internas; o tal vez provengan del entorno y se denominan "arrastre" de las fuerzas externas. Por ello resulta esencial que los administradores reconozcan la importancia de ambos tipos de fuerzas (una dualidad más importante), cuando se analizan las causas que motivan la conducta. En principio es importante señalar que enfocarse en alguna de las fuerzas y 
excluir a la otra ocasionaría diagnósticos equivocados y acciones incapaces de resolver los problemas motivacionales. Por ejemplo, un administrador supondría que el nivel de llamadas de ventas de un subalterno es bajo porque éste es flojo, cuando en realidad los incentivos que se le ofrecen no son adecuados a sus necesidades o intereses. El administrador pensaría que la causa es falta de fuerzas internas de empuje, cuando un análisis más preciso en este caso se enfocaría en las fuerzas externas de arrastre. Esta clase de interpretaciones equivocadas sobre la motivación, que son muy comunes, provocarían la pérdida de empleados potencialmente valiosos.

Asimismo, suponer que un trabajador administrativo está realizando especialmente bien su trabajo para satisfacer a su supervisor, enfatizando las fuerzas externas o de arrastre para explicar su motivación, quizá esté subestimando la importancia de las fuerzas internas de empuje. Un trabajador podría ser alguien altamente motivado sin importar el tipo de supervisión o de dirección que reciba. En ambos ejemplos, una visión más amplia de los factores motivacionales debería conducir a evaluaciones más válidas y más útiles.

A lo largo de este capítulo, examinaremos diferentes tipos de fuerzas motivacionales, con particular énfasis en lo que algunos psicólogos y otros científicos del comportamiento han establecido sobre el contenido y el proceso de motivación.

Primero, comencemos con un marco de referencia para analizar las fuentes de las fuerzas motivacionales en la situación laboral. Posteriormente, examinaremos las principales teorías conductistas de la motivación. En las últimas secciones del capítulo, centraremos nuestra atención en la manera en que los sistemas de refuerzo y el ambiente social del trabajo pueden afectar tanto la fuerza como la dirección de la motivación.

David (2011). La motivación se define como el proceso de influir en las personas para que cumplan con determinados objetivos. La motivación explica por qué ciertas personas trabajan con gran ahínco y otras no. Los objetivos, las estrategias y las políticas tienen pocas posibilidades de éxito si los gerentes y empleados no están motivados para implementar las estrategias formuladas. La función de motivación de la administración se compone de al menos cuatro componentes principales: liderazgo, dinámica de grupos, comunicación y cambio organizacional. Cuando los gerentes y empleados de una empresa se esfuerzan por alcanzar niveles altos de productividad, indica que los estrategas de la empresa son buenos líderes. Los buenos líderes 
establecen buenas relaciones con sus subordinados, son empáticos con sus necesidades y preocupaciones, representan un buen ejemplo y son confiables y justos.

Chiavenato (2012), dice que es aquello que origina una propensión hacia un comportamiento específico. Este impulso a actuar puede provocarlo un estímulo externo (que proviene del ambiente) o puede ser generado internamente en los procesos mentales del individuo. Motivación se asocia con el sistema de cognición del individuo. Aquello que origina una propensión hacia un comportamiento específico. Este impulso a actuar puede provocarlo un estímulo externo (que proviene del ambiente) o puede ser generado internamente en los procesos mentales del individuo. Motivación se asocia con el sistema de cognición del individuo.

\section{La Motivación Intrínseca}

En cualquier momento, en la persona puede generase un pensamiento (éste puede ser provocado por el razonamiento, la memoria o el subconsciente). Los pensamientos a su vez se relacionan con los sentimientos y las emociones, que terminan en una actitud. Para generarse una actitud, tanto positiva como negativa, intervienen factores como la autoimagen, las creencias, los valores y los principios éticos y morales del individuo. Si el sentido de la actitud es positivo, automáticamente se genera una excusa para realizar la acción. A esta excusa se le conoce con el nombre de motivación. Claro está que, en la acción intervienen el conocimiento, las capacidades y las habilidades del individuo, de los cuales dependerá la calidad del resultado, de acuerdo a las expectativas suyas o de los demás.

\section{Motivación Extrínseca}

Para que se genere la motivación es necesario que factores externos actúen sobre los elementos receptores (pensamiento, sentimiento y acción). Para que los factores externos sean motivadores es necesario que se genere un diferencial en la percepción de la persona. El mejor ejemplo es el salario de un empleado, como se conoce, en sí el salario no es un factor motivador, pero en el instante que hay un aumento de éste, se genera un diferencial externo que actúa sobre el pensamiento y/o sentimiento que por cierto tiempo motiva para obtener mejores resultados, si ese es el objetivo, hasta que desaparece la percepción del diferencial generado y pasa a ser un factor de mantenimiento. 


\section{Desempeño laboral}

Mora (2007), establece que el desempeño son aquellas acciones o comportamientos observados en los empleados que son relevantes para los objetivos de la organización, y que pueden ser medidos en términos de las competencias de cada individuo y su nivel de contribución a la empresa. Además, agrega, que la definición de desempeño debe sercompletada con la descripción de lo que se espera de los empleados, además de una continua orientación hacia el desempeño efectivo.

Amorós (2009). Son acciones observables dirigidas hacia una meta, donde el individuo manifiesta la voluntad y la capacidad de ejecutarla, siempre y cuando el contexto sea idóneo para alcanzarlas. Lo podemos considerar como un procedimiento estructural y sistemático para medir, evaluar e influir sobre los atributos, comportamientos y resultados relacionados con el trabajo, así como el grado de ausentismo, con el fin de descubrir en qué medida es productivo el empleado y si podrá mejorar su rendimiento futuro, que permite implantar nuevas políticas de compensación, mejora el desempeño, ayuda a tomar decisiones de ascensos o de ubicación, permite determinar si existe la necesidad de volver a capacitar, detectar errores en el diseño del puesto y ayuda a observar si existen problemas personales que afecten a la persona en el desempeño del cargo.

Las áreas de evaluación del desempeño laboral Amorós (2009), corresponden a la satisfacción al cliente, se entiende como la actitud mostrada por el trabajador para satisfacer desde el principio las necesidades de los usuarios en cuanto al desarrollo de sus funciones, o bien para resolver situaciones que a éste se le presenta durante la obtención de sus servicios o bienes, implica la disponibilidad del trabajador para responder asertivamente a las necesidades de las personas que requieren de su ayuda o conocimientos. Así mismo involucra las estrategias utilizadas por los trabajadores para satisfacer las necesidades de los clientes o el personal que tiene a su cargo. Es la disposición y actitud permanente para satisfacer y superar las necesidades y expectativas de los usuarios internos y externos. Implica otorgar un valor agregado al servicio, cumpliendo con las actividades encomendadas, con el fin de fidelizar al usuario y brindar confiabilidad y credibilidad a la organización. Trabajo en equipo, es la actitud mostrada por el trabajador de laborar en conjunto con sus compañeros así como la disponibilidad que muestra para crear, desarrollar, alcanzar y renovar creativamente 
objetivos comunes. Implica la capacidad del trabajador de desempeñar sus funciones de manera equitativa con el resto del personal de una organización. Es la capacidad de delegar funciones en caso de reconocer limitaciones personales, técnicas o de conocimiento para que éste pueda desempeñarse de manera asertiva y sea potencialmente productivo. Del mismo modo se califica la capacidad para comunicarse con los demás, a través de la expresión verbal o escrita, en cuanto a la claridad de transmisión de sus ideas hacia los demás, así como la comprensión de las ideas que se le transmiten. Es la capacidad de realizar sus actividades de manera conjunta para el logro de metas comunes, promoviendo la participación activa de cada persona. Implica desarrollar un sentido de pertenencia entre los miembros del equipo, integrando esfuerzos y destrezas de cada miembro a fin de alcanzar las metas propuestas. Logros laborales, se concibe como aquellos objetivos concretos que se fijan en un determinado periodo de tiempo a considerar cumplir las metas personales y profesionales propuestas dentro de la organización y las áreas de trabajo. Dichos logros y actividades desarrolladas son aspectos puntuales que no necesariamente se insertan en las de otras áreas de trabajo que se plantean, es decir los logros se identifican por áreas y se evalúan en relación a la calidad de la producción y el cumplimiento del tiempo establecido a desarrollarse las metas, así mismo se pueden consideran desde aspectos tales como metas de productividad, disminución de ausentismo, higiene y seguridad ocupacional las cuales son importantes para la empresa. Por tal motivo es importante que cada área de trabajo tome en cuenta los índices de productividad y desempeño por cada trabajador, con la finalidad de que se puedan efectuar cambios que posibiliten lograr el mejoramiento continuo individual y grupal de una organización.

En este aspecto se toma en cuenta también la capacidad que tiene el trabajador para comprometerse con las labores que se le asignan dentro de la empresa, es decir es la disposición que permite sentir como propios los objetivos de la Organización a fin de trabajar bajo el concepto de mejoramiento continuo en las oportunidades de mejora que se presenten. Permite guiar el comportamiento en base a normas y principios morales. Implica actuar con probidad e integridad, conduciéndose de manera coherente en base a una jerarquía de valores y buenas prácticas profesionales. Se evalúa el nivel de calificación profesional del trabajador frente al conocimiento técnico práctico de principios, normas y procesos requeridos en el ejercicio de su cargo, como resultado de 
su preparación y experiencia; así como su capacidad de análisis y evaluación profesional sobre asuntos correspondientes al ámbito de su competencia. Liderazgo, donde se entiende como la ejecutora de aquel conjunto de aspectos que determinan el correcto incremento y desarrollo de los trabajadores y de sí mismo para desempeñarse óptimamente dentro de sus funciones. Se entiende como la ejecutora de aquel conjunto de aspectos que determinan el correcto incremento y desarrollo de los trabajadores y de sí mismo. Los aspectos que se sugiere considerar son los siguientes:

- Planeación: El líder realiza este proceso mediante la organización y procesamiento de la información con la que cuenta, para planificar tareas futuras. - Delegación: Esto se refiere a que el jefe de un área de trabajo asigna autoridad a una persona para llevar a cabo actividades específicas. Si no existiese la delegación, una sola persona tendría que hacer todo y su desempeño laboral no sería el más óptimo por la cantidad de funciones a desarrollarse en un mismo tiempo.

\section{Condiciones de delegación entre trabajadores}

Coordinación: Es un proceso que consiste en integrar actividades de la misma área con el efecto de perseguir las metas de la organización con eficacia. Sin coordinación, los trabajadores perderían de vista sus papeles dentro de la organización y enfrentaría la tentación de perseguir los intereses de su departamento, a expensas de las metas de la organización.

Resolución de problemas: El desempeño del trabajador se evalúa por la capacidad del mismo para desarrollar estructuras de relaciones capaces de resolver las cuestiones difíciles y de establecer normas que faciliten el entendimiento de las alternativas de solución que éste brinda.

Capacitación y enseñanza: El trabajador a cargo de una determinada área de trabajo que ha recibido orientación y capacidades constantes tiene mayor ventajas para desempeñarse laboralmente dentro de un estándar alto de productividad, así mismo sabe que debe instruir a las personas que tiene a su cargo, pues él ha sido equipado, trasladando sus experiencias tanto buenas como malas para que sirvan de ejemplo, a su vez se procura que las personas a su cargo estén equipadas en todas las áreas que le competen para que puedan ejercer su trabajo con excelencia.

Crecimiento Personal, el desempeño laboral también se ve influenciado por el cumplimiento de aquellos objetivos de vida, totalmente personales que van en función 
no sólo del mejoramiento individual, sino que también inciden directa o indirectamente en la ejecución laboral óptima. Este aspecto podrá ser establecido únicamente por el trabajador o bien hacerlo en conjunto con su superior inmediato. Sin embargo quedará a juicio de este último con base en la posibilidad de evaluar el mejoramiento laboral del personal con su desarrollo personal.

Uzcátegui (2011), estos son los conceptos de efectividad, eficiencia y eficacia: a).Eficacia.-Grado en que se logran los objetivos y metas de un plan, es decir, cuanto de los resultados esperados se alcanzó. La eficacia consiste en concentrar los esfuerzos de una entidad en las actividades y procesos que realmente deben llevarse a cabo para el cumplimiento de los objetivos formulados. b).- Eficiencia.- Es el logro de un objetivo al menor costo unitario posible. En este caso estamos buscando un uso óptimo de los recursos disponibles para lograr los objetivos deseados. C.- Efectividad. -Este concepto involucra la eficiencia y la eficacia, es decir, el logro de los resultados programados en el tiempo y con los costos más razonables posibles. Supone hacer lo correcto con gran exactitud y sin ningún desperdicio de tiempo o dinero. De las anteriores definiciones se puede apreciar que los indicadores de efectividad y eficacia son susceptibles de ser utilizados para todo tipo de áreas de organización independientemente del carácter de su actividad, es decir, sean labores comerciales, intelectuales, de producción, de control, etc.

Téllez (2009). Los Indicadores, sostiene que los indicadores son herramientas de control que permiten monitorear los objetivos trazados, para lo que se espera que cumplan como mínimo las características reconocidas. El indicador debe proporcionar información suficiente para no sesgar su análisis, debe ser oportuno y permitir la toma de decisiones y el control efectivo.

Indicadores de Eficiencia (mide el cómo): Miden el nivel de ejecución del proceso, se concentran en el Cómo se hicieron las cosas y miden el rendimiento de los recursos utilizados por un proceso.

Indicadores de Eficacia (mide el qué): Miden el grado de cumplimiento de los objetivos propuestos, se enfocan en el Qué se debe hacer, para este indicador se deben conocer y definir los requerimientos del cliente del proceso para comparar lo que entrega el proceso contra lo que él espera.

Indicadores de Efectividad (mide el para qué): Miden la satisfacción de las necesidades. 
Uzcátegui (2011), la eficiencia y la efectividad son dos adjetivos de naturaleza cualitativa, ambos aplicables a los procesos logísticos o cualquier área en general, pues en condiciones ordinarias se propende a la optimización; lo que implica eficiencia y en condiciones extraordinarias se debe cumplir la misión aún a costa de los medios, sin llegar a convertirse en victorias pírricas; es importante entender, que la eficacia no es un defecto, pues una alta eficiencia depende de seguir estrictamente los lineamiento de la planificación, pero es conocido que la planificación debe ser flexible, pues existen variables influyentes, especialmente las del entorno que producen cambios que de no poderse actuar en ellos podrían producir el fracaso, es en estas contingencias donde la eficacia se impone. Por otra parte, la efectividad es la cuantificación del cumplimiento de la meta, no importa si ésta se logra en forma eficiente o en forma efectiva. En algunos casos, se acepta la efectividad como el logro de una meta acertadamente seleccionada en el proceso de planificación, es decir, la hipótesis que producía la solución idónea al problema o necesidad existente. Sin embargo, esto corresponde a la asertividad, palabra ésta, que no se encuentra en el diccionario.

Uzcátegui (2011), es sumamente importante entonces y entendiendo que existe una brecha entre lo establecido por la Real Academia y la usanza ordinaria a nivel gerencial y en los postgrados del área, adherir ambas realidades; lo cual puede lograse al tomar como referencia las siguientes acepciones:

Eficiencia: "Capacidad para lograr un fin empleando los mejores medios posibles". Aplicable preferiblemente, salvo contadas excepciones a personas y de allí el término eficiente.

Eficacia: "Capacidad de lograr el efecto que se desea o se espera, sin que priven para ello los recursos o los medios empleados". Esta es una acepción que obedece a la usanza y debe ser reevaluada por la real academia; por otra parte, debe referirse más bien a equipos.

Efectividad: "Cuantificación del logro de la meta". Compatible el uso con la norma; sin embargo, debe entenderse que puede ser sinónimo de eficacia cuando se define como "Capacidad de lograr el efecto que se desea".

\section{ESTRATEGIAS METODOLÓGICAS O MATERIALES Y MÉTODOS}

El estudio utilizó el método lógico deductivo consistente en la aplicación de los principios teóricos sobre la Motivación para medir la relación con el Desempeño Laboral de los 
trabajadores. La población estaba conformada por 84 trabajadores de las diferentes áreas de la UE-400 DIRES San Martín. Se tomó como muestra a la totalidad de la población. El tipo de muestreo empleado es no probabilístico, pues no se empleó fórmula. La recolección de datos se llevó a cabo por medio de encuestas aplicadas a cada uno de los trabajadores de la UE-400 DIRES-SM (para evaluar la motivación de estos); así mismo, se entregaron encuestas a los jefes de área con la finalidad de que evalúen el desempeño de sus subordinados. Para efectos del establecimiento de la relación entre las variables, se trabajó con la prueba de RhoSpearman (Posterior a la prueba de normalidad de Kolmogorov Smirnov).

\section{RESULTADOS Y DISCUSIÓN RESULTADOS}

\section{Motivación}

\section{Motivación extrínseca}

Demuestran que el $56 \%$ de los individuos evaluados se encuentra regularmente motivados, en comparación a un $31 \%$ que manifestó encontrarse desmotivado. Estos resultados se deben a que gran parte de los trabajadores se muestran indiferentes frente al hecho de que les reconozcan el logro e importancia de realizar cada uno de sus trabajos, consideran que solo en ocasiones los incentivos que reciben son adecuados, por lo que no necesariamente es un factor motivacional que contribuya con la mejora de sus labores. Sin embargo, se evidenciaron puntos importantes que estos resaltan, como el hecho de considerar que su ambiente de trabajo, así como la estructura son seguros, y que, además, el centro de trabajo se encuentra en condiciones aceptables de limpieza y de comodidad.

\section{Motivación intrínseca}

Presenta que el $56 \%$ de los trabajadores encuestados se encuentra desmotivado, un $32.1 \%$ presenta una motivación regular y solo el $11.9 \%$ se encuentra motivado; ello se debe a que en su mayoría, manifiestan sentir que el trabajo que se encuentran desarrollando, no cubren en su totalidad todos sus intereses y necesidades personales, así mismo, manifiestan que solo en ocasiones, sus compañeros y jefe inmediato, los alientan e impulsan a continuar con el logro de sus metas personales. Así mismo, una parte de los trabajadores, hacen referencia que no pueden fijarse metas laborales y 
personales por la carga laboral con la que cuentan, lo que se ve empeorada por la falta de apoyo por parte de sus superiores y compañeros.

\section{Nivel de motivación}

Establece como resultado que el 54.8\% de los trabajadores se encuentran desmotivados, ello debido principalmente a cuestiones internas (factores intrínsecos), tales como el hecho de que gran parte de los trabajadores sienten que sus necesidades no son del todo cubiertas por el trabajo que se encuentran desarrollando, o por el hecho de que no sienten que sus compañeros y jefes inmediatos les brinden el apoyo respectivo para continuar con el desarrollo de sus carreras o metas laborales y personales. Por otra parte, existen trabajadores que se muestran indiferentes frente a los factores externos como los incentivos que reciben dentro de la organización, o que los reconozcan y feliciten por lo que hacen.

\section{Desempeño laboral de los trabajadores}

Se evidencia que el $50 \%$ de los trabajadores presenta un desempeño bajo, el $35.7 \%$ un desempeño medio y solo el $14.3 \%$ un desempeño alto; estos resultados se deben a que, luego de haber realizado la tabulación de las respuestas del jefe inmediato de los trabajadores, se evidenció que las personas evaluadas carecen de un amplio conocimiento sobre todos los procedimientos que se desarrollan dentro de su unidad o área de trabajo, así mismo, se evidencia que frente a un problema determinado, este no es resuelto de manera asertiva, haciendo uso inadecuado de los recursos que se provee. Otro de los aspectos que no se evidencian en los trabajadores, es el hecho de que, una gran parte de estos se siente mejor al trabajar de manera individual que grupal, aun cuando esto conlleve al retraso de ciertos trabajos en determinados momentos. Por último, son pocos los trabajadores que se esfuerzan para lograr alcanzar las metas o actividades que aún tienen pendiente.

\section{Relación entre la motivación y el desempeño laboral}

La prueba empleada fue la de Kolmogorov-Smirnov, pues se trabajó con una muestra superior a cincuenta casos; al analizar los resultados obtenidos por dicha prueba, se evidencia que los valores sigs. Asintót. (bilateral) de cada uno de los elementos comparados (dimensiones y variables) no se encuentran normalmente distribuidos, pues se obtuvieron valores inferiores a 0.05 , siendo estos equivalentes a 0,031 - 0,042 - 0,003 y 0,005 para las dimensiones de motivación extrínseca e intrínseca y las variables 
motivación y desempeño laboral respectivamente. Por esta razón, la prueba de relación empleada es la de Rho Spearman.

Tabla 1. Prueba de normalidad de Kolmogorov-Smirnov

\begin{tabular}{llcccc}
\hline & & $\begin{array}{c}\text { Motivación } \\
\text { extrínseca }\end{array}$ & $\begin{array}{c}\text { Motivación } \\
\text { intrínseca }\end{array}$ & Motivación & Desempeño \\
\hline N & & 84 & 84 & 84 & 84 \\
Parámetros normales a,b & Media & 48,50 & 28,32 & 76,82 & 53,60 \\
& Desviación típica & 11,826 & 68,926 & 20,084 & 15,515 \\
& Absoluta & 0,158 & 0,152 & 0,199 & 0,188 \\
Diferencias más extremas & Positiva & 0,158 & 0,152 & 0,199 & 0,188 \\
& Negativa & $-0,085$ & $-0,101$ & $-0,108$ & $-0,092$ \\
Z de Kolmogorov-Smirno & & 1,445 & 1,389 & 1,825 & 1,727 \\
Sig. asintót. (bilateral) & & 0,031 & 0,042 & 0,003 & 0,005 \\
\hline
\end{tabular}

Fuente: Elaboración propia.

Prueba de Spearman entre la motivación extrínseca por desempeño, muestran que existe una relación significativa entre la motivación extrínseca y el desempeño de un total de ochenta y cuatro (84) trabajadores de la UE-400 DIRES SM, pues, el valor sig. (Bilateral) obtenido $(0,000)$ es menor a 0,050. Así mismo, dicha relación se encuentra dentro de una correlación positiva media, pues el valor del coeficiente de correlación obtenido es de 0.584 .

Prueba de Spearman entre la motivación intrínseca por desempeño, muestran la existencia de una relación significativa, pues, el valor sig., obtenido es menor a 0,05 y se encuentra a nivel de 0,000. De la misma manera, la relación existente se encuentra dentro del rango de correlación positiva muy fuerte, pues, el valor del coeficiente de correlación, es igual a 0,752 .

Al realizar la comparación de los resultados de la variable motivación y la variable desempeño laboral, se evidencia una relación significativa a nivel de 0,000, así mismo, dicha relación se encuentra dentro del rango de una calificación positiva considerable, pues el coeficiente de correlación arrojado por la prueba de Rho Spearman es de 0,671 
Tabla 2 Prueba de Spearman entre la motivación por desempeño

\begin{tabular}{cclcc}
\hline & & \multicolumn{2}{c}{ Motivación } & Desempeño \\
\hline \multirow{4}{*}{ Rho de Spearman } & Coeficiente de correlación & 1,000 &, $671^{* *}$ \\
& & Motivación & & 0,000 \\
& & Sig. (bilateral) & 84 & 84 \\
& & Coeficiente de correlación & $0,671^{* *}$ & 1,000 \\
& \multirow{2}{*}{ Desempeño } & Sig. Bilateral & 0,000 & \\
& & $\mathrm{~N}$ & 84 & 84 \\
\hline
\end{tabular}

Fuente: Elaboración propia

**. La correlación es significativa al nivel 0,01 (bilateral).

\section{DISCUSIÓN}

La motivación laboral constituye el impulso de satisfacer una determinada necesidad por medio de diferentes actividades dentro de un marco de trabajo. Estas afirmaciones se ven respaldas por lo mencionado por Chiavenato (2012), quien manifiesta en sus libros, que las personas se ven incentivadas por medio de diferentes factores, los cuales pueden ser resumidos en dos grandes grupos: Los factores externos (motivación extrínseca) y los factores internos (motivación intrínseca). Al evaluar cada uno de estos factores por medio de un cuestionario a los trabajadores de la UE-400 DIRES -SM, se llegó a establecer que gran parte de los trabajadores (54.8\%), presentan una motivación laboral baja, debido fundamentalmente a que no cuentan con adecuados factores motivacionales de índole interno, y porque, además, no se ven muy entusiasmados con las formas de como los motivan dentro de la institución. Estos resultados guardan relación con el trabajo realizado por Delgado (2010), ya que este investigador determina que la motivación es un factor importante dentro de una organización, y que de encontrarse en situaciones negativas (tal y como es el caso en ambas investigaciones), presenta una repercusión en contra de los objetivos de la institución. Cabe señalar que Delgado, hace hincapié al señalar que, dentro de su institución, el factor económico (retribución), ha dejado de ser el principal factor motivacional para los trabajadores, tal y como es el caso en la presente investigación.

El desempeño laboral constituye las actividades, así como las diferentes formas que aplica un trabajador para desarrollar una determinada actividad dentro de un tiempo determinado. Su evaluación dentro de la organización es de suma importancia, pues, contribuye al establecimiento de estándares y a la toma de decisiones respecto a las 
acciones a desarrollar con los trabajadores. Muchos son los autores que buscan una forma razonable y optima de evaluar el desempeño laboral, tal y como es el caso de Amorós (2009), de quien, luego de haber definido al desempeño como un conjunto de acciones observables en un trabajador hacia la consecución de una meta establecida, se identificó tres aspectos importantes a evaluar en los trabajadores de la UE-400 DIRES San Martín: "la eficiencia, la eficacia y la fectividad". Los resultados obtenidos indican que el $50 \%$ de los colaboradores presentan un bajo nivel de desempeño, pues, carecen de un amplio conocimiento sobre todos los procedimientos que se desarrollan dentro de su unidad o área de trabajo, así mismo, se evidencia que, frente a un problema determinado, este no es resuelto de manera asertiva, haciendo uso inadecuado de los recursos que se provee, entre otros problemas. Al comparar los resultados con el trabajo de Torres (2011), se identifica que el desempeño también representó un problema dentro de la realidad de la cooperativa evaluada por el investigador, sin embargo, el principal problema de la institución fue el desconocimiento de los objetivos y metas, que conllevó a retrasar los resultados esperados en la cooperativa.

Luego de haber analizado cada una de las variables de forma independiente, se llegó a establecer el grado de relación entre las mismas, por medio de la aplicación de pruebas estadísticas como la prueba de normalidad de Kolmogorov Smirnov y la prueba de correlación de Rho Spearman, con los cuales se llegó a demostrar la existencia de una relación significativa a nivel de 0,000 entre la motivación laboral y el desempeño laboral, y luego de llevar a cabo la prueba de contrastación de la hipótesis, se llegó a aceptar que: La relación entre la motivación y el desempeño laboral de los trabajadores de la UE - 400-DIRES SM en el periodo 2014 significativa. Estos resultados guardan relación con los encontrados por Delgado (2010), quien luego de haber evaluado la relación entre la motivación laboral y el desempeño laboral, establece que gran parte de los problemas que giran en torno al desempeño de los colaboradores, son ocasionados por un bajo nivel de motivación en los trabajadores.

\section{CONCLUSIÓN O CONSIDERACIONES FINALES}

Se estableció que los trabajadores de la UE-400 DIRES San Martín, presentan un nivel bajo de motivación, siendo el factor intrínseco, la dimensión que presentó índices más bajos que el factor extrínseco; ello se debió a que los colaboradores se muestran indiferentes hacia aspectos como los incentivos económicos que puedan recibir dentro 
de la institución, ya que le entregan mayor importancia a los aspectos relacionados con su propia persona, como la fijación y cumplimiento de sus metas personales.

Alrededor del 50\% de los colaboradores, presentaron niveles bajos de desempeño, debido a su falta de conocimiento sobre la totalidad de las actividades que se desarrollan dentro de su unidad o área de trabajo, o por emplear más recursos de los necesarios, y en el peor de los casos, por no cumplir con las metas establecidas por una falta de esfuerzo.

Por último, se concluye la investigación afirmando la existencia de una relación entre la motivación y el desempeño laboral de los trabajadores de la UE-400 DIRES San Martín, pues luego de comparar los resultados de ambas variables mediante algunas pruebas estadísticas como lo es Rho Spearman, se estableció una relación a nivel de 0,000, llegándose a aceptar la hipótesis alterna.

\section{LISTA DE REFERENCIAS}

Amorós, E. (2009). Organizaciones. (1 ${ }^{\mathrm{a}}$ ed). Perú: Águila Ediciones.

Arnoletto, E. (2001) “Comportamiento y Desarrollo Organizacional”. Material de estudio del Curso de Especialización de Gestión Integral de la Calidad. UTN, Facultad Regional Córdoba.

Arnoletto, E. (2005) La Gestión Organizacional en la Administración Pública. Primera edición. UCC: Córdoba.

Brewer C. (1979) Política, Estado y Administración Pública. Ediciones Conjuntas. Caracas. pp. $45-84$.

Chiavenato, I. (2009). Administración de recursos humanos: el capital humano en las diversas organizaciones. México: Editorial MC Graw Hill.

Chiavenato, I. (2012). “Cultura Organizacional”. Edit. Pearson Educación. México. 3ra edición.

Delgado, M. (2010). La motivación laboral y su incidencia en el desempeño laboral: un estudio de caso, Universidad Central de Venezuela Facultad de Humanidades y Educación.

García, M. (1977) Las Transformaciones del estado Contemporáneo. Alianzas Editorial Madrid.

Gorostiaga, R. (1996) Transparencia en la gestión de gobierno, CITAF-OEA.

Hayes, B. (1999) Cómo medir la satisfacción de los: cliente. Oxford Inglaterra, Segunda 
edición.

Mejía, Y. (2012) "Evaluación del desempeño con enfoque en las competencias laborales",

Universidad Rafael Landíva, Guatemala, (Tesis de Pregrado) recuperado de http://biblio3.url.edu.gt/Tesario/2012/05/43/Mejia-Yessika.pdf.

Mino, E. (2014) “Correlación entre clima organizacional y desempeño en los trabajadores

del restaurante de parrillas Marakos 490 del departamento de Lambayeque", Universidad Católica Santo Toribio de Mogrovejo, departamento de Lambayeque.

Mora, C. (2008) Calidad Humana. Recopilado el 08 de octubre, de http.77www.gestiopolis.com/canales7/rrhh/calidad-humana.htm

Pineda, L. (2004). Importancia del clima organizacional y el rendimiento laboral en los empleados de una Institución Pública de Administración de Justicia. Guatemala.

Robbins, S. (2009). Comportamiento organizacional teoría y práctica. 1996. p 214. http: ://www.marketing-xxi.com/teorias-de-la-motivacion-90.htm,102003

Rojas moya J. (2007). Gestión por procesos para mejorar la atención del usuario en los establecimientos de salud del sistema nacional de salud, www.eumed.net/libros/2007a/

Saturno J. Pedro: Cómo diseñar una evaluación sobre la calidad asistencial en atención primaria, Unidad docente de medicina preventiva y salud pública, Universidad de Murcia. España

Téllez, L. (2009). Universidad de Quindío. Sistema Integrado de Gestión. Generalidades de la Medición en el Sector Público- Los Indicadores. Recopilado el 19 de marzo,

de

http://web2.uniquindío.edu.co/dep/plandes/documentos/sig/capacitaciones/ indicadores.pdf.

Torres, J. (2011). La evaluación del desempeño laboral y su incidencia en los resultados del rendimiento de los trabajadores de la cooperativa de ahorro y crédito OSCUS Ltda. Ambato. 2010. Ecuador.

Uzcátegui, J. (2011) Dimensiones del Desempeño. Águila Editores. Colombia. 